\title{
Barium Strontium Titanate Thick-Films : Dependency between Dielectric Performance and their Morphology
}

\author{
Giere, A.; Zhou, Xiandong; Paul, F. et al.
}

(2008)

$\begin{array}{ll}\text { DOI (TUprints): } & \text { https://doi.org/10.25534/tuprints-00013398 } \\ \text { License: } & \text { only the rights of use according to UrhG } \\ \text { Publication type: } & \text { Article } \\ \text { Division: } & 18 \text { Department of Electrical Engineering and Information Technology } \\ \text { Original source: } & \text { https://tuprints.ulb.tu-darmstadt.de/13398 }\end{array}$




\title{
Barium Strontium Titanate Thick-Films: Dependency between Dielectric Performance and their Morphology
}

\author{
By A. Giere, X. Zhou, F. Paul, M. Sazegar, Y. Zheng, H. Maune, J. R. Binder, and R. Jakoby
}

\begin{abstract}
The paper is focused on the influence of the morphology of barium strontium titanate (BST) thick-films on their effective dielectric properties. Therefore two BST thick-films are made with a minor deviation in sinter temperature, and hence their mean grain size of the films and their sintering necks. By modeling the meso-structure using a Nonlinear 3D Finite-Difference Time-Domain field solver, the assumed effect of the morphology on the effective dielectric properties are confirmed. The simulation results show the
\end{abstract} potential for a further improvement of the dielectric tunability of BST thick-films.

Index Terms -morphology, thick-film, ferroelectric, composite modeling

\section{Introduction}

Ferroelectric ceramics such as barium strontium titanate (BST) are promising candidates for tunable devices, because the permittivity of ferroelectric materials decreases when an external E-field is applied to them. BST can be engineered in thin-film or thick-film process to possess the required properties in terms of permittivity, dielectric loss and tunability. BST thin-films have been fabricated through physical vapour deposition [1], chemical vapour deposition [2] and pulsedlaser deposition [3], which can be expensive and time consuming. Compared to the thin-films, screen printed BST thickfilms are cost effective but have a typical larger dielectric loss factor in radio frequency (RF) region. However, the dielectric loss of BST can be reduced though doping with iron and fluorine. The doping resulted defect and charge compensation can affect the dielectric properties of BST, especially its tunability and dielectric losses [4].

Process parameters such as sintering temperature in fabricating ceramic thick-film always have influences on the morphology of the samples. It was reported, that the sintering time and temperature affected the grain size in another ceramic system [5]. The grain size and grain boundary relatedeffect of $\mathrm{BaTiO} 3$ and $\mathrm{SrTiO} 3$ nano-grain ceramic on dielectric properties have been discussed by Buscaglia [6] and Petzelt [7]. However, the functional properties of thick- and thin-film are usually different from those of their bulk counterparts, due to the mesoscopic size effect associated with film morphology [8]. In this paper, we introduce an approach to describe the correlation between the thick-film morphology of doped $\mathrm{Ba}_{06}$ $\mathrm{Sr}_{0.4} \mathrm{TiO}_{3}$ and their dielectric properties such as permittivity and tunability in RF region by means of a NonLinear 3D Finite-Difference-Time-Domain (NL3D-FDTD) electromagnetic field solver which is adapted to the nonlinear behavior of the used ferroelectric material $[9,10]$.

\section{Characterization of the BST thick-films morphology}

$\mathrm{Ba}_{0.60} \mathrm{Sr}_{0.40} \mathrm{Ti}_{0.99} \mathrm{Fe}_{0.01} \mathrm{O}_{3-\delta} \mathrm{F}_{\delta}$ powders were synthesized from barium acetate, strontium acetate, and titanium-(IV)-isopropoxide through sol-gel process. Iron nitrate and trifluoroacetic acid as dopants were added into the reactants. Then the sol was spray-dried into metal-organic precursor and the ceramic powders were obtained though calcination of the precursor at $900^{\circ} \mathrm{C}$. The chemical analysis with ion chroma- tography in laboratory of H.C. Starck showed that the substance amount fraction of fluorine is $0.46 \%$.

The thick-films were screen printed with applying BST powders, vehicle and dispersant contained paste onto polycrystalline alumina substrate (Rubalit710, CeramTec).

In order to adjust the grain size in thick-films, they were sintered in dry air at temperatures $1180^{\circ} \mathrm{C}$ and $1200^{\circ} \mathrm{C}$ for one hour. The processing and morphology characterization of the BST thick-film are made at the Institute for Materials Research III of the Karlsruhe Research Center, Karlsruhe, Germany

The film microstructures were investigated through scanning electron microscope (SEM, Supra 55, Zeiss) and image analysis software AnalySIS Pro from Olympus in term of grain size distribution. Four SEM images in top view of each sample were used in the image analysis to determine the grain size. The diameter of 200 grains at each image was measured.

Fig. 1a and Fig. 1b show the microstructures of the BST grains in thick-film sintered at $1180^{\circ} \mathrm{C}$ and at $1200^{\circ} \mathrm{C}$ for one hour respectively. The temperature effect on the grain growth of BST is obvious. In the sample, which is sintered at $1180^{\circ} \mathrm{C}$
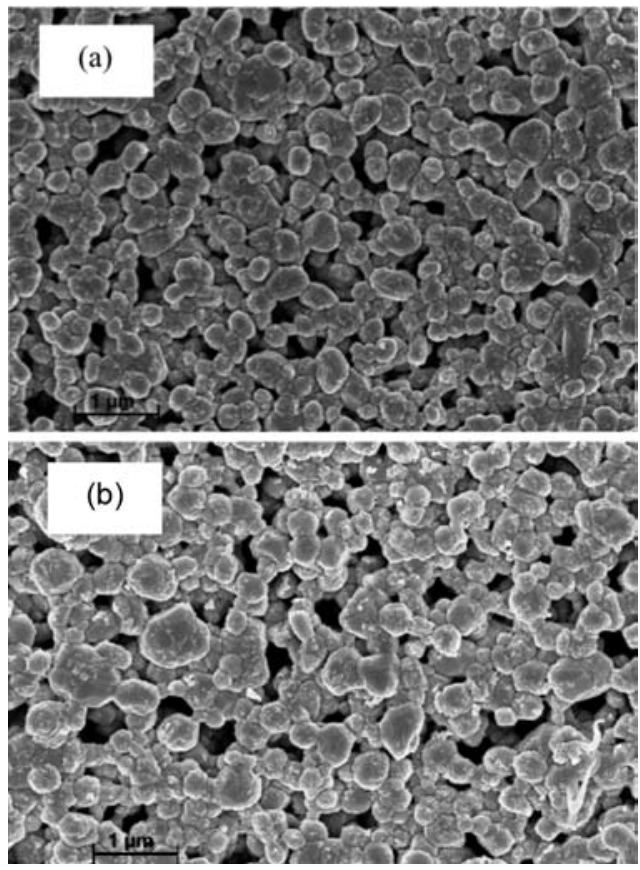

Fig. 1: SEM images of sintered BST thick-film at (a) $1180^{\circ} \mathrm{C}$ and (b) $1200^{\circ} \mathrm{C}$ 


\begin{tabular}{|l|l|l|l|}
\hline Name & sintering temperature & mean value & standard deviation \\
\hline BST-A & $1180^{\circ} \mathrm{C}$ & $0,34 \mu \mathrm{m}$ & $0,11 \mu \mathrm{m}$ \\
\hline BST-B & $1200^{\circ} \mathrm{C}$ & $0,38 \mu \mathrm{m}$ & $0,12 \mu \mathrm{m}$ \\
\hline
\end{tabular}

smaller grain sizes and smaller sintering necks are observable. It can be indicated in the result of image analysis quantitatively.

The grain size distribution histogram of both thick-films (Fig. 2) shows the effect of the sintering temperature quantitatively. Most of the grains sintered at $1180^{\circ} \mathrm{C}$ are in the $300 \mathrm{~nm}$ to $350 \mathrm{~nm}$ fraction, during majority of the grains sintered at $1200^{\circ} \mathrm{C}$ are settled between $350 \mathrm{~nm}$ and $400 \mathrm{~nm}$. In Tab. 1, the measured mean value and standard deviation of the grain size are given. From this table, it can be easily seen that the $20^{\circ} \mathrm{C}$ higher temperature has made a grain growth in the thick-film.

The size of sintering necks is difficult to determine in the SEM images quantitatively. But it can be observed that the grains in Fig. 1b are well connected and the sintering necks are stronger. As expected, the increasing temperature enlarged not only the grain size, but also the sintering necks.

\section{Dielectric characterization of BST thick-films}

For the characterization of the tuning field dependent permittivity, coplanar waveguide (CPW) structures are realized on top of both BST thick-films. The metallization of the CPW is processed by a single lithography step on a $\mathrm{Cr} / \mathrm{Au}$ evaporated seed layer and strengthened by an Au galvanic step. The cross-section and the geometry of the remaining CPWs are shown in Fig. 3.

The tuning voltage dependent transmission line characteristics of the CPWs are determined by temperature controlled on-wafer S-parameter measurements using a vector network analyzer by sweeping the tuning voltage in a range from $0 \mathrm{~V}$ to $100 \mathrm{~V}$. From the transmission line the complex capacitance per unit-length is computed using an inductance per unit-length calculated by Conformal Mapping (CM) method. Based on the remaining complex capacitance value, the permittivity of the BST film is extracted using the iterative characterization method using a 2D-Finite-Difference Time-Domain method (2D-FDTD) based electromagnetic field solver adapted to the nonlinear material [10]. This method considers the inhomogeneous tuning field distribution in the BST thick-film and further the related inhomogeneous tuned permittivity.

The resulting tuning field dependent permittivity and the tunability are shown in Fig. 4 for both BST thick-films and shows the different dielectric properties due to the changed

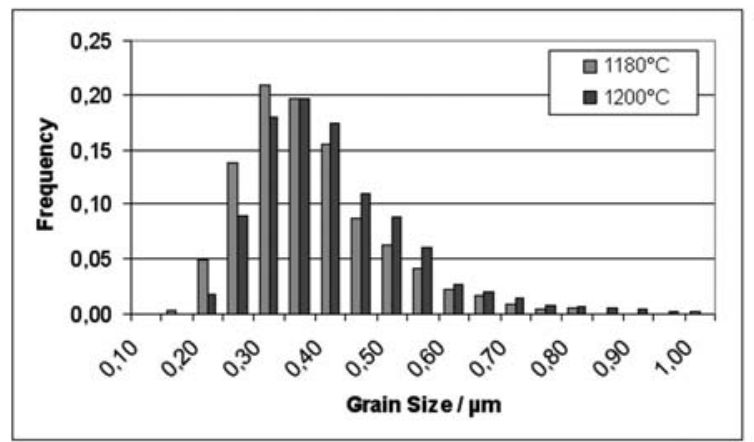

Fig. 2: Influence of the sintering temperature on the grain size distribution

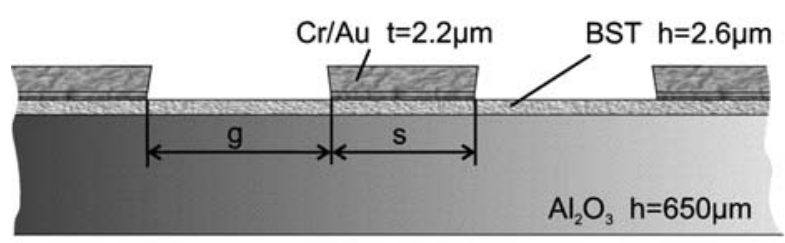

Fig. 3: CPW cross-section on top of screen-printed BST thick-film. On substrate BST-A / BST-B the gapwidth is $\mathrm{g}=8.36 \mu \mathrm{m} / 8.05 \mu \mathrm{m}$ and the metal stripwidth is $S=20.36 \mu \mathrm{m} / 20.83 \mu \mathrm{m}$ respectively.

sinter temperature. The extracted untuned permittivity of the thick-film BST-A is $\varepsilon_{\mathrm{r}}=202$ and $\varepsilon_{\mathrm{r}}=249$ from BST-B where the tunability at $10 \mathrm{~V} / \mu \mathrm{m}$ changes from $\tau=32.8 \%$ to $\tau=36.2 \%$. To study the origin of different dielectric properties we model the morphology of the porous ceramic in the following section.

\section{Approach to model the meso-structure of porous thick-films}

For evaluation of the dependency of the meso-structure on the resulted effective nonlinear dielectric properties, a mesoscopic model of the structure is required. Due to the restricted amounts of analytic models being able to consider the effect of the meso-scopic structure in porous ferroelectric thick-films, first we show the limitation of the existing composite based models. Thereby we assume that the thick-films meso-structure is a composite based on ferroelectric material and air having a relative permittivity of $\varepsilon_{\mathrm{r}}=1$. The porosity $p$ of the thick-film thereby equivalent to $(1-q)$ where $q$ is the proportion of included ferroelectric material to the whole volume.

Three analytic models for mixed ferroelectric and linear dielectric based composites based on composites models using pure linear dielectrics are presented in [11]. These serial stacked layers, columnar composite and spherical inclusion models are shown in Fig. 5. Assuming an inclusion of ferroelectric material into a linear dielectric cube, the placement of the ferroelectric material effects dramatically the effective dielectric properties of the, so called cubic unit-cell.

For the untuned state $(E=0)$ of the effective material, the models response are equal to the pure composite models based on only linear dielectrics. An effective tuning field is used to consider a tuned permittivity of the ferroelectric material. To be able to compute the effective material properties all three models are using a constant effective electric tuning field-strength which is homogeneous distributed inside both dielectrics. In case of the spherical inclusion model (Fig. 5c) these assumptions neglecting the inhomogeneous electric field in the sphere and further in the unit-cell. In addition the spherical inclusion model is limited to only small inclusions of ferroelectric material into the linear dielectric based unit-cell $(q<<1)$.

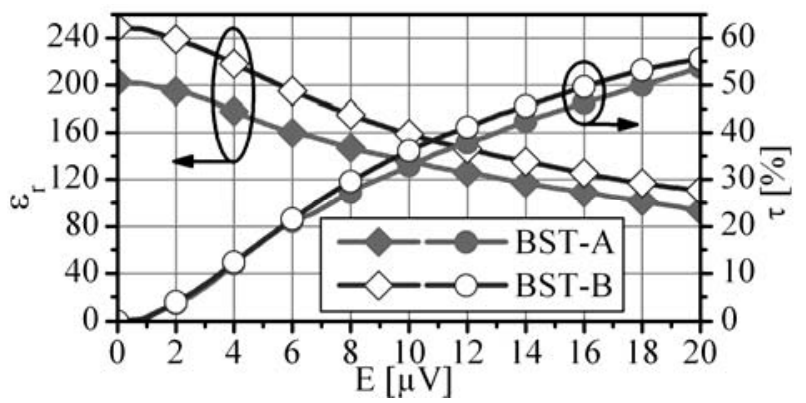

Fig. 4: Extracted permittivity and tunability of both BST thick-films using the 2D-FDTD based extraction method [10] 


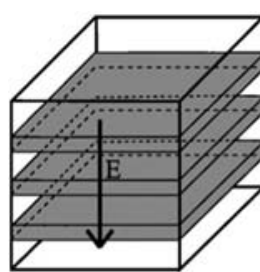

a)

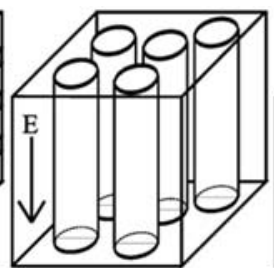

b)

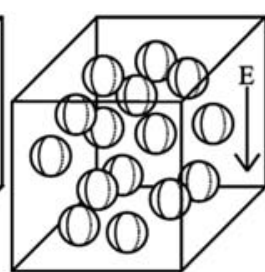

c)
Fig. 5: a) Serial stacked layer model of a dielectric composite. b) Columnar composite model. c) Spherical inclusion model [11].

In [12] and [13] a further extension for modeling ferroelectric / linear dielectric composites based on the Modified Effective Medium Approximation (MEMA) is presented which describe the inclusion of linear dielectric and ferroelectric spheres into a cube. This model overcome the limitation of $q<<1$ from the spherical inclusion model in Fig. 5c. But also this model is only able to consider a homogeneous distribution of the electric tuning field inside the both dielectric and ferroelectric spheres and assuming the same sphere diameter for the dielectric and ferroelectric material.

To overcome the limitation of the analytic models, and to be able to consider the inhomogeneous distribution of the electric tuning field, an electromagnetic field solver based on the Finite-Difference Time-Domain method in 3D is adapted to be able to simulate a more realistic meso-structure of nonlinear materials than possible by using the analytic composite models. By using the NL3D-FDTD solver $[9,10]$ we are able to compute the effective dielectric properties from a capacitance simulation having the dielectric unit-cell between two perfect conducting electrodes. In detail the meso-structure modeled and simulated by NL3D-FDTD is shown in Fig. 6b, while in Fig. 6a a zoom into the meso-structure of a porous BST thick-film is shown for comparison. The model based on an air filled unit-cell with inclusion of spheres representing BST grains having a variable diameter $d_{\text {grain }}$. Each sphere is connected to the six direct neighboring spheres by a cylinder, representing the sintering neck having a diameter of $d_{\text {neck }}$ where the distance between the centers of the adjacent spheres is $s_{\text {grain }}$ (see Fig. 6c). This meso-structure model implicit only connection between the different BST grains directed in $0^{\circ}$ and $90^{\circ}$ to the applied electric field, and uses further only an average BST grain diameter compared to the real thick-film meso-structure.

From material characterization of BST thick-films up to now only the effective dielectric properties of the film are known. Therefore we use dielectric properties from BST bulk ceramic measurements lowered in relative permittivity to consider defects in the Perovskite crystal at the sintering necks and grain boundaries and the used iron doping which lowers in addition the permittivity. The chosen material parameters are listed in Table 2.

Table 2: For simulation used dielectric properties for BST grain and sintering neck.

\begin{tabular}{|l|l|l|l|}
\hline Frequency & untuned permittivity $\varepsilon_{\mathrm{r}}(0)$ & tan $\delta$ & tunability $\tau$ \\
\hline $30 \mathrm{GHz}$ & 400 & 0,05 & $25,2 \% @ 5 \mathrm{~V} / \mu \mathrm{m}$ \\
\hline
\end{tabular}

\section{Simulation of a thick-films meso-structure}

For studying of the effect of the meso-structure, the distance between two grains will be constant at $s_{\text {grain }}=500 \mathrm{~nm}$ where the diameter of the BST grain is changed between $d_{\text {grain }}=350 \mathrm{~nm}$ to $500 \mathrm{~nm}$ and the diameter of the cylinder representing the sintering neck varied between $d_{\text {neck }}=0 \mathrm{~nm}$ and $400 \mathrm{~nm}$.

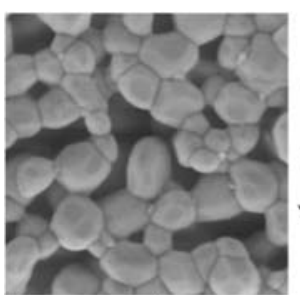

a)

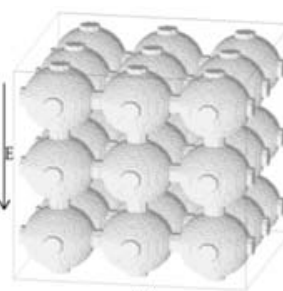

b)

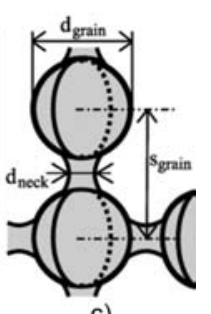

c)
Fig. 6: a) Zoom into a real structure of a porous BST thick-film. b) Unit-cell for the simulation of the meso-scopic effect consists of BST grains connected with sintering necks. c) Parameters of the grain / sintering neck structure in the unit-cell.

The convergence of the effective dielectric properties the NL3D-FDTD simulation requires mesh sizes of $10 \mathrm{~nm}$ which corresponds to a mesh size in the order of $\lambda_{0} / 10^{6}$ when exciting at $30 \mathrm{GHz}$. Because of the extreme dense mesh and the related computation time, the NL3D-FDTD simulations are performed at the CSC cluster in Frankfurt am Main, Germany.

The resulted untuned permittivity from simulation is shown in Fig. 7 with the varied diameters of the grain and the sintering neck.

The result shows a strong dependency of the untuned permittivity on the diameter of the sintering neck where the diameter of the grain did not affect the untuned permittivity $\varepsilon_{\mathrm{r}}$ (0) significantly. The surface is generated by interpolating the 78 simulation results.

The dependency of the effective untuned dielectric properties can partly be explained by changing porosity of the simulated meso-structure as shown in Fig. 8.

Comparing the porosity with the resulted untuned effective permittivity, it results in a lower dependency of the grain diameter than of the sintering neck diameter on the unit-cells $\varepsilon_{\mathrm{r}}(0)$.

To be able to evaluate the effective properties for applied electric tuning field strength, the NL3D-FDTD solver calculates first the distribution of the electrostatic tuning field in the unit-cell when applying an tuning voltage to the capacitance which corresponds to an effective tuning field strength of $E_{\text {eff }}=5 \mathrm{~V} / \mu \mathrm{m}$. In a second step before applying the RF excitation, the inhomogeneous distributed electric tuning field is applied to the nonlinear characteristic of the BST material to compute the local field dependent permittivity. In Fig. 9 the resulted local adapted relative permittivity is shown for $d_{\text {grain }}=500 \mathrm{~nm}$ and $d_{\text {neck }}=0 \mathrm{~nm}$.

After further computation the tuned effective permittivity at $30 \mathrm{GHz}$ of the unit-cell the tunability of the meso-structure can be determined. For evaluation the meso-structures tunability $\tau_{\text {meso }}$ in respect to the tunability of the BST bulk material

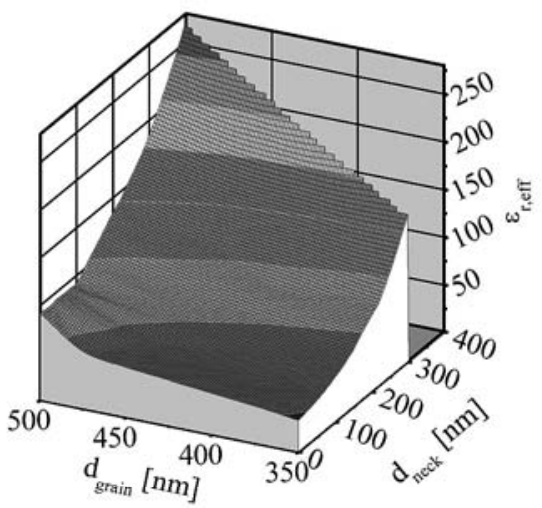

Fig. 7: Effective untuned permittivity of the simulated meso-structure in dependency of the grain diameter and diameter of the sintering necks 


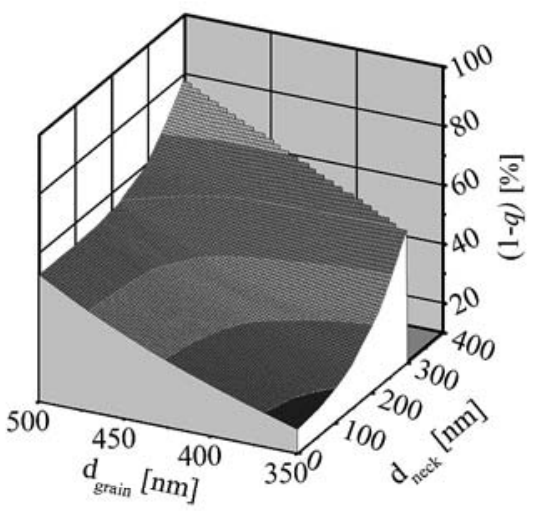

Fig. 8: Concentration of the porosity $(1-q)$ of the meso-structure when changing the grain and sintering neck diameter.

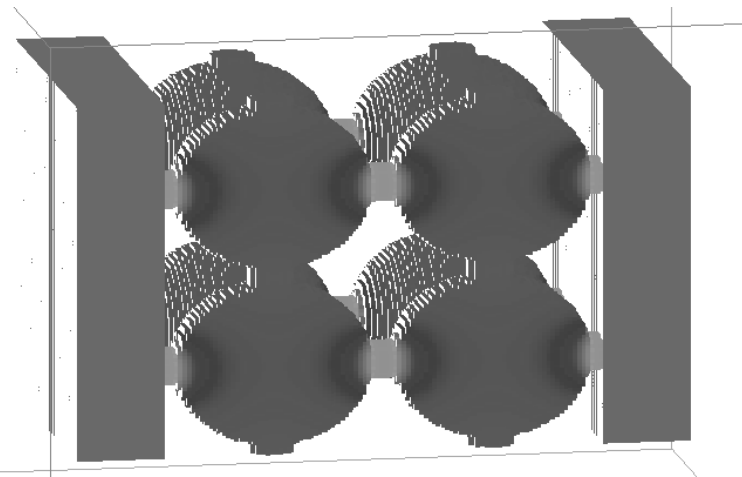

Fig. 9: Permittivity distribution when applying an effective tuning field of $5 \mathrm{~V} / \mu \mathrm{m}$ on the unit-cell by the electrodes on the left and right. Close to the sintering necks along the serial path the relative permittivity drops down to $\varepsilon_{\mathrm{r}} \approx 133$ where inside the grains and at the connections perpendicular to the E-field the permittivity is close to the untuned value $\left(\varepsilon_{\mathrm{r}} \approx 400\right)$.

$\tau_{\text {bulk }}$, the material tuning efficiency factor $\chi_{\text {meso }}$ defined by eqn. 1 is used.

$\chi_{\text {meso }}=\frac{\tau_{\text {meso }}}{\tau_{\text {bulk }}}$

As shown in Fig. 10, the meso-structure is able to increase the tuning efficiency using the provided material tunability up to $\chi_{\text {meso }} \approx 155 \%$ when the diameter of the sintering neck gets close to $0 \mathrm{~nm}$. In addition the efficiency decreases continuously down to $100 \%$ by increasing the diameter of the sintering neck towards the diameter of the grains. :

The increased efficiency can be explained by comparing the path (grain - sintering neck - grain) of the electric field with in serial connected capacitances where the smallest capacitance

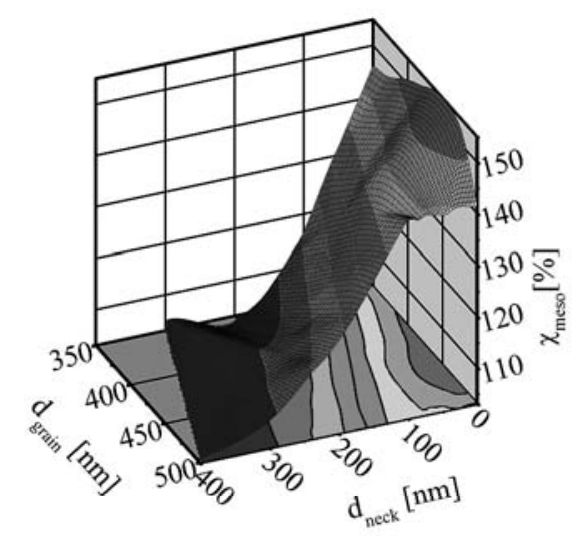

Fig. 10: Material tuning efficiency factor $\chi_{\text {meso }}$ in dependency of the two sweeped parameters. is the ones with the biggest impact on the whole performance. The capacitance associated with the sintering neck between two grains, is in this serial connection the smallest ones. Due to a superelevation of the tuning field surrounding the sintering necks this area is the highest tuned ones having the lowest permittivity (Fig. 9).

\section{Discussion and Conclusion}

To investigate the relation between the process dependent morphology of ferroelectric thick-films and their dielectric properties, two screen-printed BST thick-films with different sintering temperatures are realized and characterized in the $\mathrm{GHz}$ range. Due to this temperature difference the morphology changes significantly measured by the mean grainsize of the film. In addition a change of the sintering necks diameter is observed. This change in morphology results in a relative permittivity variation of $19 \%$ and a tunability variation of $9.4 \%$ with respect to BST-A. By using a model for simulation based on the thick-films meso-structure, we are able to model the influence of grain size and sintering necks. The dependency of the simulation result matches quite well with the observed dependency between morphology and measured effective dielectric properties. Due to the complexity of the real meso-structure, the model is only able to represent parts of the meso-structure.. However, the simulation has shown that a change of the grain size from $350 \mathrm{~nm}$ to $390 \mathrm{~nm}$, which approximates the change in BST thick-film morphology, results in a $16.8 \%$ increased permittivity and a $6.4 \%$ increased tunability assuming a constant diameter of the sintering necks of $100 \mathrm{~nm}$.

Using an appropriate morphology the effective dielectric properties of the BST thick-film can be further improved. The diameter of the sintering necks is hereby the most sensitive parameter. By optimizing the meso-structure a high material tuning efficiency of $>150 \%$ can be archived with a reduced diameter of the sintering neck.

The observed strong dependency of the sintering temperature on the morphology and further on the effective dielectric properties shows thereby the need and promising potential of a precise control of the sintering parameters.

\section{References}

[1] N. K. Pervez and P. J. Hansen and R. A. York, High tunability barium strontium titanate thin films for $\mathrm{rf}$ circuit applications, Applied Physics Letters, vol. 85, [2004], pp. 4451-4453

[2] Battiston, G. A. and Gerbasi, R. and Carta, G. and Marchetti, F. and Pettinari, C. and Rodriguez, A. and Barreca, D. and Maragno, C. and Tondello, E., Innovative second-generation Ba and Sr precursors for chemical vapor deposition of $\mathrm{Ba1}-\mathrm{xSrxTiO} 3$ thin films, Journal Of The Electrochemical Society, vol. 153, [2006], pp. F35 - F38

[3] Gong, J. and Cheng, J. and Zhu, W. and Yu, S. and Wu, W. and Meng, $\mathrm{Z}$., Improvement in dielectric and tunable properties of $\mathrm{Fe}$-doped $\mathrm{Ba} / \mathrm{sub} 0.6 / \mathrm{Sr} / \mathrm{sub} 0.4 / \mathrm{TiO} / \mathrm{sub} 3 /$ thin films grown by pulsed-laser deposition, Ultrasonics, Ferroelectrics and Frequency Control, IEEE Transactions on, vol. 54, [2007], pp. 2579-2582

[4] F. Paul, Dotierte Ba0.6Sr0.4TiO3-Dickschichten als steuerbare Dielektrika (Pulversynthese und dielektrische Eigenschaften), Dissertation

[5] Brunckova, H. and Medvecky, L. and Mihalik, J., Effect of sintering conditions on the pyrochlore phase content in PMN-PFN ceramics prepared by sol-gel process, Journal Of The European Ceramic Society, vol. 28, [2008], pp. 123-131

[6] Buscaglia, V. and Buscaglia, M. T. and Viviani, M. and Mitoseriu, L. and Nanni, P. and Trefiletti, V. and Piaggio, P. and Gregora, I. and Ostapchuk, T. and Pokorny, J. and Petzelt, J., Grain size and grain boundary-related effects on the properties of nanocrystalline barium titanate ceramics, Journal Of The European Ceramic Society, vol. 26, [2006], pp. 2889-2898

[7] Petzelt, J. and Ostapchuk, T. and Gregora, I. and Savinov, M. and Chvostova, D. and Liu, J. and Shen, Z. J., Grain boundary effects on dielectric, infrared and Raman response of $\mathrm{SrTiO} 3$ nanograin cera- 
mics, Journal Of The European Ceramic Society, vol. 26, [2006], pp. $2855-2859$

[8] Du, Quangen and Li, JiangYu and Nothwang, William and Cole, Melanie W., The dielectric behavior of polycrystalline ferroelectric films with fiber textures, Acta Materialia, vol. 54, [2006], pp. $2577-$ 2587

[9] A. Giere, P. Scheele, C. Damm and R. Jakoby, "Optimization of Uniplanar Multilayer Structures using Nonlinear Tunable Dielectrics", Proc. 35th European Microwave Conf. 2005, pp. 561-564, 2005

[10] A. Giere, P. Scheele, Y. Zheng and R. Jakoby, "Characterization of the Field-Dependent Permittivity of Nonlinear Ferroelectric Films Using Tunable Coplanar Lines", IEEE Microwave and Wireless Components Letters, vol. 17 (2007) pp. 442-444.

[11] A. Tagantsev, V. Sherman, K. Astafiev, J. Venkatesh and N. Setter, "Ferroelectric Materials for Microwave Tunable Applications", Journal of Electroceramics, vol. 11, pp. 5-66, 2003

[12] V. O. Sherman, A. K. Tagantsev, N. Setter, D. Iddles and T. Price, "Ferroelectric-dielectric tunable composites", Journal of Applied Physics, AIP, vol. 99, pp. 074104, 2006

[13] V. O. Sherman, A. K. Tagantsev and N. Setter, "Model of a lowpermittivity and high-tunability ferroelectric based composite", Applied Physics Letters, AIP, vol. 90, pp. 162901, 2007

This work was supported in part by the German Research Foundation (DFG) Grant JA 921/8-1, by the DFG Research Training Group 1037

"Tunable integrated components in microwave technology and optics (TICMO)," and by the Frankfurt Center for Scientific Computing.
Andre Giere, Xianghui Zhou, Mohsen Sazegar, Yuliang Zheng, Holger Maune, Rolf Jakoby

Microwave Engineering

Technische Universität Darmstadt

Merckstrasse 25

64289 Darmstadt / Germany

Fax: +49 6151/16-4322

E-mail: giere@hf.tu-darmstadt.de

Xianghui Zhou, Joachim R. Binder Institute for Materials Research III

Forschungszentrum Karlsruhe,

Hermann-von-Helmholtz-Platz 1 ,

76344 Eggenstein-Leopoldshafen / Germany

Fax: +49 7247/82-4612

E-mail: Xianghui.Zhou@imf.fzk.de

\section{Florian Paul}

IMTEK,

University of Freiburg,

Georges-Koehler-Allee 102

79110 Freiburg / Germany
Frequenz

$62(2008)$

$3-4$ 